\title{
UTILIZAÇÃO DE TÉCNICAS MULTIVARIADAS NA CLASSIFICAÇÃO DE FASES DE CRESCIMENTO DE Leucaena leucocephala (LAM.) DE WIT.
}

\author{
Leonardo Mendes de Lima Júnior ${ }^{1}$, Rinaldo Luiz Caraciolo Ferreira ${ }^{2}$, José Antônio Aleixo da Silva ${ }^{3}$, \\ Eufrázio de Souza Santos ${ }^{4}$, Mário de Andrade Lira Júnior ${ }^{5}$, Ivan Ferraz ${ }^{6}$

\begin{abstract}
${ }^{1}$ Estatístico, M.Sc., Depto. de Estatística e Informática, UFRPE, Recife, PE, Brasil - leomljunior@ hotmail.com
${ }^{2}$ Eng. Florestal, Dr., Depto. de Ciência Florestal, UFRPE, Bolsista CNPq, Recife, PE, Brasil - rinaldo@ dcfl.ufrpe.br

${ }^{3}$ Eng. Agrônomo, Ph.D., Depto. de Ciência Florestal, UFRPE, Pesquisador 1D do CNPq, Recife, PE, Brasil - aleixo@dcfl.ufrpe.br

${ }^{4}$ Estatístico, Dr., Depto. de Estatística e Informática, UFRPE, Recife, PE, Brasil - eufrazio@ deinfo.ufrpe.br

${ }^{5}$ Eng. Agrônomo, Ph.D., Depto. de Agronomia, UFRPE, Recife, PE, Brasil - mario.lira@ depa.ufrpe.br

${ }^{6}$ Eng. Agrônomo, M.Sc., Estação Exp. de Caruaru, Instituto Agronômico de Pernambuco (IPA), Recife, PE, Brasil - ivan@ipa.br
\end{abstract}

Recebido para publicação: 05/06/2008 - Aceito para publicação: 21/11/2008

\begin{abstract}
Resumo
Objetivou-se identificar as fases de crescimento da leucena (Leucaena leucocephala (Lam.) de Wit.) na presença ou não de composto orgânico, por meio da utilização das técnicas multivariadas. Os dados foram obtidos de um experimento instalado na Estação Experimental de Caruaru (PE) do Instituto Agronômico de Pernambuco (IPA). Neste estudo, consideraram-se, além das 19 medições em altura (em m), outras 18 medições, interpoladas através do modelo de Weibull e por interpolação linear, dividindo-se em 2 grupos, segundo a presença ou não de composto de resíduo orgânico. A análise fatorial permitiu a redução da dimensionalidade das medições, em função de três fatores, com variação explicada de $94,60 \%$ e $94,30 \%$, nos tratamentos sem e com composto orgânico, respectivamente. Dos fatores retidos, obtiveram-se os escores, que foram submetidos à análise de agrupamento k-médias, sendo o número de grupos escolhidos previamente, para de $\mathrm{k}$ variando de $3 \mathrm{a}$ 10. Os grupos de melhores classificações foram verificados por meio de análise discriminante, com $95,2 \%$ e $95,1 \%$ de classificação correta. Identificou-se que as plantas que receberam o composto orgânico tiveram as maiores alturas, em média. A análise gráfica permitiu a comparação entre os dados que tiveram tratamento com composto orgânico e sem composto orgânico, conforme os grupos, em sua fase de crescimento. Com a análise fatorial, foi possível representar as fases de crescimento da leucena na presença ou não de composto orgânico.
\end{abstract}

Palavras-chave: Análise de agrupamento; análise discriminante; análise fatorial.

\begin{abstract}
The use of multivariate analysis on classification of the Leucaena leucocephala (Lam.) de Wit. growth phases. The aim of this work was to identify the growth phases of leucena (Leucaena leucocephala) in presence or absence of organic compound residue, by using of the multivariate analysis techniques. The data set was obtained through an experiment performed at the Experimental Station of CaruaruPE of the Institute of Agricultural Research - IPA. Besides 19 actual measures of height, additional 18 values were used, obtained by interpolation of best fit curves to the Weibull model, as well as by the linear interpolation, for two groups: according to presence or absence of organic compound residue. The factor analysis was used to reduce the dimensionality of measured data to three factors with explaining $94.60 \%$ and $94.30 \%$ of the total variation for the treatments with and without organic compound, respectively. The resulting factor scores were subjected to k-means cluster analysis, using previously selected, number of groups $\mathrm{k}$ from 3 to 10 . The discriminant analysis was then employed to verify the efficiency of clustering of the best classification groups, which was found to be $95.2 \%$ and $95.1 \%$ of correct classification for the two treatments. The study showed that the leucena trees fertilized with the organic compound presented greater heights. The graphical analysis allowed the comparison between the data treatment with and without organic compound treatment, according to groups, in growth phase. With the fatorial analysis was possible to represent the phases of growth of the leucena in the presence or not of composed organic.
\end{abstract}

Keywords: Cluster analysis; discriminant analysis; factor analysis. 


\section{INTRODUÇÃO}

A cada dia se expandem as áreas sem vegetação arbórea, para utilização agrícola e/ou pecuária, Mas pouca preocupação tem havido com áreas de florestas, ou com a recuperação de áreas degradadas através de reflorestamento. Entre as espécies mais promissoras das leguminosas para esse fim, encontrase a leucena (Leucaena leucocephala (Lam.) de Wit.), que pertence à tribo Mimosae, subfamília Mimosideae, de boa adaptação em todas as regiões brasileiras na produção de florestas energéticas, contribuindo por completo com produtividade e ajudando a estabelecer e enriquecer o solo (OLIVEIRA et al., 2004).

A leucena cresce bem nas regiões tropicais, em diferentes tipos de ambientes, apresentando um sistema radicular profundo, alta taxa de crescimento, resistência às pragas e doenças, simbiose com bactérias fixadoras de nitrogênio e tolerância à seca (FREITAS et al., 1991). Mesmo havendo grande interesse dos produtores pela leucena na região Nordeste, sua utilização ainda é bastante restrita, talvez devido à falta de tradição no uso dessa leguminosa (SILVA, 1992).

Drumond (2001) afirmou que a leucena é uma espécie de rápido crescimento, chegando a crescer até 3 metros de altura no primeiro ano. Seiffert;Thiago (1983), contudo, relataram que o crescimento da leucena nos três primeiros meses é bastante lento, o que leva à necessidade de fertilização, visando maior crescimento dessa espécie.

A compreensão e a predição do crescimento das árvores em um povoamento florestal vem sendo tema de grande interesse por profissionais que atuam na pesquisa e na gestão dos recursos florestais (MAESTRI et al., 2003).

As principais tarefas atribuídas ao profissional da ciência florestal são entender como ocorre o crescimento das árvores de um povoamento, qual tratamento silvicultural adotar e prever qual será a época de corte e a produtividade do plantio (CLUTTER et al. 1983; DAVIS; JOHNSON, 1987).

O acompanhamento do crescimento permite construir uma base de dados capaz de proporcionar estimativas do crescimento florestal, produção, desbastes e outras práticas silviculturais (SCOLFORO, 1993).

$\mathrm{Na}$ implantação de um povoamento florestal, o manejador precisa levar em consideração, entre outros fatores, as condições gerais de crescimento e produção (sítios) dentro de uma mesma área. Nesse caso, a classificação em áreas homogêneas permite inferir sobre a capacidade produtiva de classe de sítio e fundamentar a elaboração e execução dos planos de manejo.

Atualmente, a análise de crescimento é realizada com a utilização de métodos cada vez mais avançados, dada a facilidade encontrada de acesso a computadores de alto desempenho e a software especializado em tais análises (MENDES et al., 2006). Um dos maiores interesses em pesquisa em biometria florestal é desenvolver modelos de crescimento e produção para a predição do crescimento de uma floresta ou de uma árvore individual (ROSSI et al., 2007).

Na classificação de sítio, há necessidade de utilização de várias variáveis e, consequentemente, a aplicação de técnicas estatísticas apropriadas para o estudo das inter-relações entre os diversos fatores, evidenciando-se a importância da aplicação de técnicas estatísticas multivariadas.

A análise estatística multivariada possibilita avaliar a estrutura de covariância de um vetor aleatório composto pelas variáveis correspondentes às características estudadas. Várias são as técnicas para essa análise: análise de componentes principais, análise fatorial e análise de correlação canônica, entre outras.

Segundo Burton et al. (1991), as técnicas estatísticas multivariadas têm sido amplamente empregadas em estudos envolvendo, simultaneamente, variáveis de clima, solo, relevo, vegetação e geologia na classificação de áreas com cobertura florestal. Essas técnicas são utilizadas com objetivos básicos de ordenamento, visando determinar a influência de fatores do meio na composição e produtividade do local, e de agrupamento, com o propósito de classificação.

Segundo Anderson (1984), existem, basicamente, duas formas de classificar as análises multivariadas: as que permitem extrair informações a respeito da independência entre as variáveis que caracterizam cada elemento, tais como análise fatorial, análise de agrupamento, análise canônica, análise de ordenamento multidimensional e análise de componentes principais; e as que permitem extrair informações a respeito da dependência entre uma ou mais variáveis ou uma com relação à outra, tais 
como análise de regressão multivariada, análise de contingência múltipla, análise discriminante e análise de variância multivariada.

O objetivo deste trabalho foi identificar as fases de crescimento da leucena na presença ou não de composto orgânico, empregando-se as técnicas estatísticas multivariadas da análise fatorial de agrupamento e discriminante.

\section{MATERIAL E MÉTODOS}

\section{Dados utilizados}

Foram utilizados dados de altura de um povoamento de Leucaena leucocephala de Wit. (Hawai), variedade K8, de procedência australiana. O experimento foi implantado em dezembro de 1989, na Estação Experimental da Empresa Pernambucana de Pesquisa Agropecuária (IPA) em Caruaru (PE), considerando 3 fatores com diferentes níveis, o que resultou em 24 tratamentos repetidos inicialmente 24 vezes (MEUNIER, 1991).

As alturas foram mensuradas em 19 ocasiões: aos 11, 37, 65, 98, 128, 159, 189, 220, 255, 287, $318,361,479,571,935,1277,1466,1837$ e 3256 dias após o plantio. Foram estudadas as fontes de variação do uso do composto de resíduo urbano: níveis de adubação com superfosfato simples (18\% de $\mathrm{P}_{2} \mathrm{O}_{5}$ ) e estirpes de bactérias fixadoras de nitrogênio atmosférico.

Além disso, foram obtidos novos valores por interpolação, de forma a reduzir os grandes espaçamentos para aproximadamente 120 dias (entre 3 e 4 meses). Com isso, os dados que continham as 19 medições receberam 18 medições adicionais, totalizando 37 medidas para o estudo, de acordo com as novas datas, a idade da planta correspondente (em mês) e os dias após o plantio.

\section{Métodos estatísticos}

Interpolação de medidas com o modelo de Weibull

A interpolação entre as medidas foi estimada por meio do modelo matemático de crescimento de Weibull ajustado por Brito et al. (2007), para os tratamentos com composto orgânico (Eq. 1) e sem composto orgânico (Eq. 2):

$$
\begin{gathered}
W f=W i-0,041556412 \cdot\left(e^{4,837531361 . T f^{0,907562809}}-e^{4,837531361 \cdot T i^{0,907562809}}\right) \text { Eq. } 1 \\
W f=W i-0,078534725 .\left(e^{5,520878931 . T f^{0,755432332}}-e^{5,520878931 . T i^{0,755432332}}\right) \text { Eq.2 }
\end{gathered}
$$

Em que: $W_{f}=$ a altura ou comprimento final;

$W_{i}=$ a altura ou comprimento inicial observado;

$T_{f}=$ tempo final das medições das alturas de leucena;

$T_{i}=$ tempo inicial das medições das alturas de leucena.

Em casos em que a aplicação das equações usadas por Brito et al. (2007) extrapolou a altura final das plantas, considerou-se o incremento periódico, com interpolações lineares.

Vale ressaltar que as interpolações dentro da faixa de variação da idade foram realizadas a partir dos dados utilizados neste trabalho, o que permitiu criar um conjunto de dados com características próximas aos dados originais sem comprometimento das análises estatísticas aplicadas (NETER et al., 1996; DRAPER; SMITH, 1998).

Análise fatorial

Após as interpolações das medições, foi aplicada a análise fatorial, visando à redução da dimensionalidade das medições originais e identificação dos fatores mais relevantes ao modelo (ou seja, aqueles que melhor descrevem o comportamento da leucena ao longo do tempo), bem como classificar e detectar grupos que representem sua fase de crescimento.

Os fatores mais significativos foram extraídos conforme seu percentual de explicação da variabilidade de cada fator e, ainda, considerando o Critério de Kaiser (KAISER, 1958), que admite como importante o fator cujo autovalor correspondente é maior ou igual a 1. O método de estimação das cargas 
Fatoriais foi o das componentes principais, usando-se a matriz de correlação R. Esse método fornece os pares de autovalor e autovetor $\left(\hat{\lambda}_{\mathrm{i}}, \hat{\mathrm{e}}_{\mathrm{i}}\right), i=1, \mathrm{~K}, p$ da matriz de correlação $\mathrm{R}$, com $\hat{\lambda}_{1} \geq \hat{\lambda} 2 \geq \mathrm{K} \geq \hat{\lambda} p$, e as combinações lineares $\mathrm{Y}_{\mathrm{i}}=\underline{\hat{\mathrm{e}}}_{\mathrm{i}}^{\prime} \underline{\mathrm{X}} \quad i=1, \mathrm{~K}, p$ são as componentes principais correspondentes. $\mathrm{E}$ bem conhecido que $\mathrm{V}\left(\mathrm{Y}_{1}\right) \geq \mathrm{V}\left(\mathrm{Y}_{2}\right) \geq \mathrm{K} \geq \mathrm{V}\left(\mathrm{Y}_{\mathrm{p}}\right)$.

A partir desses elementos, estima-se a matriz de cargas fatoriais para os $\mathrm{m}$ fatores considerados mais importantes $L=\left\{\lambda_{i j}\right\}_{p \times m}$, conforme Johnson; Wichern (1998):

$$
\mathrm{L}=\left\{\sqrt{\hat{\lambda}_{1}} \underline{\mathrm{e}}_{1} ; \sqrt{\hat{\lambda}_{2}} \underline{\mathrm{e}}_{2} ; \mathrm{L} ; \sqrt{\hat{\lambda}_{\mathrm{m}}} \underline{\mathrm{e}}_{\mathrm{m}}\right\}=\hat{\mathrm{P}} \hat{\Lambda}^{1 / 2}
$$

Em que: $\hat{P}=$ matriz $\left(\begin{array}{lll}p & x & m\end{array}\right)$ dos autovetores amostrais (dos $m$ fatores mais importantes); $\hat{\Lambda}=$ matriz diagonal $(m \times m)$ dos autovalores correspondentes.

Então as cargas fatoriais são $1_{i j}=\hat{e}_{i j} \sqrt{\hat{\lambda}_{i}}$, em que $i=1,2, \ldots, p$ e $j=1,2, \ldots$, m. A relação entre a variância específica da variável i e a sua comunalidade é dada por $\hat{\psi}_{i}=1-\hat{h}_{i}^{2}=1-\sum_{j=1}^{m} \hat{1}_{i j}^{2}$. Assim, quanto maior a comunalidade, maior será a importância da variável no espaço fatorial.

O número de fatores retidos no modelo são os que apresentaram autovalores maiores ou iguais a um (KAISER, 1958). Logo, a proporção da variação explicada pelo j-ésimo fator comum obtida é:

$$
\frac{\hat{\lambda}_{\mathrm{j}}}{\sum_{\mathrm{j}=1}^{\mathrm{m}} \hat{\lambda}_{\mathrm{j}}}=\frac{\hat{\lambda}_{\mathrm{j}}}{\mathrm{p}}, j=1, \mathrm{~K}, m \text {. }
$$

A fim de interpretar os fatores, buscou-se uma estrutura mais simples, e para isso usou-se uma rotação ortogonal nos eixos coordenados. A rotação foi do tipo varimax. Os escores fatoriais foram obtidos usando-se o método da regressão, como uma combinação linear dos fatores que sofreram a rotação e das medições padronizadas, conforme Manly (1997):

$$
\underline{F}^{*}=\left(\underline{G^{\prime}} \underline{G}\right)^{-1} \underline{G}^{\prime} \underline{X}
$$

Em que: $\underline{F}^{*}=$ vetor de dimensão $m$ dos escores fatoriais;

$\underline{G}=$ vetor de dimensão $p$ das cargas fatoriais rotacionadas;

$\underline{X}=$ vetor de dimensão $p$ de médias dos caracteres da k-ésima família (medições padronizadas).

Análise de agrupamento pelo método das k-médias

$\mathrm{O}$ método das k-médias foi aplicado de forma a permitir que objetos deixem um grupo e se associem a outro, de modo que esses reposicionamentos sucessivos possam contribuir para melhorar o resultado final.

Para aplicar esse método de agrupamento, foi necessário definir, a priori, o vetor central dos agrupamentos, ou centroides, inserindo os objetos mais próximos a eles. A partir do agrupamento obtido, buscou-se avaliar a hipótese da solução encontrada pelo agrupamento.

O algoritmo básico para um número inicial de k-agrupamentos foi aplicado conforme descrito por Afif; Clark (1996): a) Os dados foram divididos em k agrupamentos iniciais, definidos previamente; b) Calculou-se a média, ou centroide, em cada um dos k-agrupamentos; c) Para um dado indivíduo, foi calculada sua distância para cada um dos k-centroides; d) Se o individuo estivesse mais perto do centroide de seu próprio agrupamento, permaneceria no próprio agrupamento, caso contrário seria deslocado para o grupo cujo centroide estivesse mais perto dele. O processo foi repetido para todos os indivíduos, até que não fosse feita nenhuma troca dos elementos para outros agrupamentos.

Análise discriminante

$\mathrm{Na}$ análise discriminante se utilizou a função discriminante de Anderson (1984), considerando que se tinha uma observação x e desejava-se classificá-la em uma dentre g populações, sendo g $>2$. 
Considerando que a densidade associada a cada população $\pi_{\mathrm{i}}$ possuía distribuição normal multivariada de parâmetros $\mu_{i}$ e $\Sigma_{i}(i=1,2, \mathrm{~K}, g)$ e que matrizes de covariância de todas as populações são iguais ( $\Sigma i=\Sigma, i=1,2, \ldots, g)$, a função discriminante foi obtida por: $D i(\mathbf{x})=\hat{\lambda}_{i}{ }_{i} \mathbf{x}-\frac{1}{2} \hat{\lambda}_{i}{ }_{i}{ }_{i}+\ln (p i)$, em que: $\quad \hat{\lambda}_{i}=S_{c}^{-1} \bar{x}_{i}, \quad S_{c}=\frac{\sum_{i=1}^{g}(n i-1)}{n-g}=$ variância combinada estimada, e $\bar{x} i=\frac{1}{n i} \sum_{j=1}^{n i} x i j, i=1,2, \mathrm{~K}, g=$ média de cada grupo $\mathrm{g}$.

A regra de decisão quanto à população de $\mathrm{x}$ consistiu em classificar $\mathrm{x}$ em $\pi_{\mathrm{i}}$ se e somente se: $D i(\mathbf{x})=\operatorname{Max}(D 1(\mathbf{x}), D 2 i(\mathbf{x}), \mathrm{K}, D g(\mathbf{x}))$, para $\mathrm{g}>2$.

Todo o processamento dos dados e o ajustamento dos modelos foi realizado através do software estatístico Statistical Package for Society Sciences, versão 13.0 (SPSS, 2004).

\section{RESULTADOS E DISCUSSÃO}

\section{Análise dos indivíduos na ausência de composto orgânico}

O modelo fatorial ajustado teve $94,60 \%$ da variabilidade total explicada pelos três fatores, por meio da rotação ortogonal varimax. As cargas fatoriais entre os fatores e as medições das alturas tiveram valores acima de 0,67 , não sendo necessário o descarte de medida (Tabela 1).

Conforme a tabela 1 , pode-se observar que a fase inicial de crescimento das plantas é explicada pelo fator 3, com explicação de 5,40\% de sua variância. Já a fase juvenil é descrita pelo fator 2 , com $13,79 \%$ de explicação da variância. E finalmente a fase senil pode ser descrita pelo Fator 1, o qual explica $75,41 \%$ da variância total.

Usando 3 funções discriminantes, os agrupamentos de melhor classificação foram os de três e de cinco grupos, ambos com 95,2\% de percentual de classificação correta. Em seguida se tem o agrupamento com 6 grupos, com 93,3\% de probabilidade de classificação correta (Tabela 2).

$\mathrm{Na}$ idade de 0,4 a 3,3 meses, verifica-se que o grupo 2 teve maior crescimento nesaa fase, variando de $0,87 \mathrm{~m}$ a $1,01 \mathrm{~m}$, em média, enquanto que o grupo 3 apresentou as menores alturas, que variaram de $0,51 \mathrm{~m}$ a $0,62 \mathrm{~m}$ na sua média (Tabela 3 ).

No período de 4,3 a 38,6 meses, ou seja, do $4^{\circ}$ mês do $1^{\circ}$ ano ao $3^{\circ}$ mês do $3^{\circ}$ ano de crescimento da planta, o grupo 1 obteve maior taxa de crescimento, com variação, em média, de $1,32 \mathrm{~m}$ a $3,91 \mathrm{~m}$,

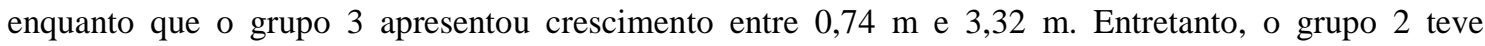
diferença a maior em relação aos grupos 1 e 3, com amplitude de 2,67 m na média (Tabela 3).

$\mathrm{Na}$ fase do $7^{\circ}$ mês do $3^{\circ}$ ano ao $12^{\circ}$ mês do $9^{\circ}$ ano, que corresponde a 42,6 a 108,5 meses, o grupo 2, com variação de $3,95 \mathrm{~m}$ a $5,54 \mathrm{~m}$, foi o de maior crescimento em altura nesse período, seguido do grupo 1, que variou de 4,0 $\mathrm{m}$ a 5,03 $\mathrm{m}$ na média. $\mathrm{O}$ grupo 3 foi o de menor crescimento, em média: de $3,42 \mathrm{~m}$ a 4,86 m. O grupo 8 apresentou maior crescimento, com variação de $0,95 \mathrm{~m}$ no $1^{\circ}$ mês a $1,34 \mathrm{~m}$ no $3^{\circ}$ mês de idade (Tabela 4 ).

$\mathrm{Na}$ fase que vai do $4^{\circ}$ mês do $1^{\circ}$ ano ao $3^{\circ}$ mês do $3^{\circ}$ ano após o plantio, o agrupamento com 8 grupos, que variou entre $1,77 \mathrm{~m}$ a $4,57 \mathrm{~m}$, teve maior desenvolvimento em altura, seguido do grupo 9 , com variação de $1,58 \mathrm{~m}$ a 4,65 $\mathrm{m}$ na média. $\mathrm{O}$ grupo de menor crescimento da leucena nesse período foi o grupo 7, com 34 plantas agrupadas e variação de $1,01 \mathrm{~m}$ a 2,70 $\mathrm{m}$ em média.

$\mathrm{Na}$ idade do $7^{\circ}$ mês do $3^{\circ}$ ano ao $12^{\circ}$ mês do $9^{\circ}$ ano, observa-se que o grupo 7 teve crescimento inferior aos demais, concluindo-se que esse agrupamento foi o de menor crescimento em todas as fases da leucena, com variação média de 2,74 a $3,91 \mathrm{~m}$, embora no $1^{\circ}$ mês de medição já possuísse altura média de $0,87 \mathrm{~m}$ (Tabela 4). 
O grupo de maior crescimento em altura foi o grupo 3 , com 8 plantas agrupadas, embora na $1^{\text {a }}$ fase este mesmo grupo tenha variado menos que o grupo 8 , de $0,89 \mathrm{~m} \mathrm{a} 1,18 \mathrm{~m}$. Na $3^{\mathrm{a}}$ fase de crescimento, as alturas variaram de 5,38 $\mathrm{m}$ a $6,92 \mathrm{~m}$ em média. Em comparação com o agrupamento com 3 grupos, observa-se que as maiores alturas médias foram mais discriminadas no $2^{\circ}$ agrupamento (figuras $1 \mathrm{a}$ e $1 b)$.

Tabela 1. Matriz de cargas fatoriais, comunalidades e variâncias específicas segundo a idade da Leucaena leucocephala (Lam.) de Wit, obtidos na rotação varimax, no tratamento sem composto.

Table 1. Matrix of factorial loadings, communalities and specific variance, according to the age of Leucaena leucocephala (Lam.) de Wit, obtained in varimax rotation for treatment without residue compound.

\begin{tabular}{|c|c|c|c|c|c|}
\hline \multirow{2}{*}{ Idade (meses) } & \multicolumn{3}{|c|}{ Fator } & \multirow{2}{*}{ Comunalidade } & \multirow{2}{*}{ Variância específica } \\
\hline & 1 & 2 & 3 & & \\
\hline 0,4 & $-0,0081$ & 0,1367 & 0,9164 & 0,8585 & 0,1415 \\
\hline 1,2 & 0,0424 & 0,2106 & 0,9605 & 0,9686 & 0,0314 \\
\hline 2,2 & 0,0849 & 0,2923 & 0,9371 & 0,9708 & 0,0292 \\
\hline 3,3 & 0,1332 & 0,5158 & 0,8051 & 0,9320 & 0,0680 \\
\hline 4,3 & 0,1882 & $\mathbf{0 , 7 5 5 2}$ & 0,5149 & 0,8709 & 0,1291 \\
\hline 5,3 & 0,2470 & 0,8391 & 0,3563 & 0,8921 & 0,1079 \\
\hline 6,3 & 0,3264 & 0,8645 & 0,2574 & 0,9201 & 0,0799 \\
\hline 7,3 & 0,3459 & 0,8784 & 0,2220 & 0,9405 & 0,0595 \\
\hline 8,5 & 0,3859 & 0,8749 & 0,2082 & 0,9577 & 0,0423 \\
\hline 9,6 & 0,4019 & 0,8751 & 0,1920 & 0,9642 & 0,0358 \\
\hline 10,6 & 0,4106 & $\mathbf{0 , 8 7 3 7}$ & 0,1903 & 0,9682 & 0,0318 \\
\hline 12,0 & 0,4322 & 0,8670 & 0,1885 & 0,9740 & 0,0260 \\
\hline 16,0 & 0,4622 & 0,8491 & 0,1813 & 0,9675 & 0,0325 \\
\hline 19,0 & 0,5743 & 0,7440 & 0,1554 & 0,9074 & 0,0926 \\
\hline 23,1 & 0,5923 & 0,7392 & 0,1533 & 0,9206 & 0,0794 \\
\hline 27,2 & 0,6284 & 0,7184 & 0,1461 & 0,9324 & 0,0676 \\
\hline 31,2 & 0,7129 & 0,6092 & 0,0933 & 0,8881 & 0,1119 \\
\hline 34,9 & 0,7277 & 0,6052 & 0,0900 & 0,9040 & 0,0960 \\
\hline 38,6 & 0,7457 & 0,5939 & 0,0851 & 0,9161 & 0,0839 \\
\hline 42,6 & 0,7665 & 0,5684 & 0,0732 & 0,9160 & 0,0840 \\
\hline 46,6 & 0,7774 & 0,5582 & 0,0723 & 0,9212 & 0,0788 \\
\hline 48,9 & 0,8193 & 0,4883 & 0,0699 & 0,9147 & 0,0853 \\
\hline 52,9 & 0,8250 & 0,4823 & 0,0713 & 0,9183 & 0,0817 \\
\hline 56,9 & 0,8346 & 0,4729 & 0,0696 & 0,9250 & 0,0750 \\
\hline 61,2 & 0,9332 & 0,3206 & 0,0713 & 0,9788 & 0,0212 \\
\hline 65,2 & 0,9351 & 0,3188 & 0,0721 & 0,9813 & 0,0187 \\
\hline 69,1 & 0,9384 & 0,3147 & 0,0713 & 0,9847 & 0,0153 \\
\hline 73,0 & 0,9418 & 0,3086 & 0,0711 & 0,9873 & 0,0127 \\
\hline 77,0 & 0,9442 & 0,3034 & 0,0714 & 0,9887 & 0,0113 \\
\hline 80,9 & 0,9459 & 0,2988 & 0,0718 & 0,9892 & 0,0108 \\
\hline 84,8 & 0,9469 & 0,2949 & 0,0721 & 0,9888 & 0,0112 \\
\hline 88,8 & 0,9475 & 0,2913 & 0,0722 & 0,9878 & 0,0122 \\
\hline 92,7 & 0,9475 & 0,2893 & 0,0727 & 0,9867 & 0,0133 \\
\hline 96,6 & 0,9475 & 0,2869 & 0,0729 & 0,9853 & 0,0147 \\
\hline 100,6 & 0,9473 & 0,2847 & 0,0731 & 0,9838 & 0,0162 \\
\hline 104,5 & 0,9471 & 0,2826 & 0,0732 & 0,9823 & 0,0177 \\
\hline 108,5 & 0,9219 & 0,2677 & 0,0835 & 0,9286 & 0,0714 \\
\hline \% Variância explicada & 75,4092 & 13,7881 & 5,4025 & - & - \\
\hline
\end{tabular}


Tabela 2. Resultado da análise discriminante, segundo percentual de classificação correta dos grupos, para a Leucaena leucocephala (Lam.) de Wit, no tratamento sem composto orgânico.

Table 2. Results from discriminant analysis, according to the correct classification percent of groups, for Leucaena leucocephala (Lam.) de Wit, in treatment without organic compound.

\begin{tabular}{lc}
\hline Número de grupos & Percentual de classificação correta \\
\hline $\mathbf{3}$ & $\mathbf{9 5 , 2 \%}$ \\
4 & $90,0 \%$ \\
5 & $92,6 \%$ \\
6 & $93,3 \%$ \\
7 & $92,6 \%$ \\
8 & $92,2 \%$ \\
$\mathbf{9}$ & $\mathbf{9 5 , 2 \%}$ \\
10 & $91,8 \%$ \\
\hline
\end{tabular}

Tabela 3. Médias e desvios padrões das alturas da Leucaena leucocephala (Lam.) de Wit, em agrupamento com 3 grupos, de acordo com a idade, para o tratamento sem composto orgânico.

Table 3. Mean and standard deviation for height in Leucaena leucocephala (Lam.) de Wit, clustering for three groups, according to the age in treatment without organic compound.

\begin{tabular}{|c|c|c|c|c|c|c|}
\hline \multirow{2}{*}{$\begin{array}{l}\text { Idade } \\
\text { (meses) }\end{array}$} & \multicolumn{2}{|c|}{ Grupo 1} & \multicolumn{2}{|r|}{ Grupo 2} & \multicolumn{2}{|c|}{ Grupo 3} \\
\hline & Média & Desvio padrão & Média & Desvio padrão & Média & Desvio padrão \\
\hline 0,4 & 0,78 & 0,16 & 0,87 & 0,17 & 0,51 & 0,15 \\
\hline 1,2 & 0,85 & 0,14 & 0,93 & 0,15 & 0,54 & 0,14 \\
\hline 2,2 & 0,89 & 0,15 & 0,96 & 0,15 & 0,57 & 0,13 \\
\hline 3,3 & 1,00 & 0,18 & 1,01 & 0,16 & 0,62 & 0,14 \\
\hline 4,3 & 1,32 & 0,28 & 1,16 & 0,24 & 0,74 & 0,20 \\
\hline 5,3 & 1,57 & 0,39 & 1,30 & 0,31 & 0,90 & 0,27 \\
\hline 6,3 & 2,01 & 0,51 & 1,63 & 0,42 & 1,25 & 0,38 \\
\hline 7,3 & 2,39 & 0,62 & 1,94 & 0,49 & 1,58 & 0,40 \\
\hline 8,5 & 2,66 & 0,69 & 2,18 & 0,55 & 1,79 & 0,47 \\
\hline 9,6 & 2,72 & 0,70 & 2,24 & 0,56 & 1,85 & 0,48 \\
\hline 10,6 & 2,74 & 0,71 & 2,26 & 0,55 & 1,87 & 0,49 \\
\hline 12,0 & 2,81 & 0,72 & 2,35 & 0,57 & 1,94 & 0,50 \\
\hline 16,0 & 2,89 & 0,73 & 2,46 & 0,58 & 2,03 & 0,53 \\
\hline 19,0 & 3,11 & 0,79 & 2,81 & 0,72 & 2,26 & 0,62 \\
\hline 23,1 & 3,37 & 0,79 & 3,07 & 0,72 & 2,54 & 0,62 \\
\hline 27,2 & 3,54 & 0,81 & 3,28 & 0,73 & 2,74 & 0,63 \\
\hline 31,2 & 3,70 & 0,82 & 3,59 & 0,77 & 3,09 & 0,72 \\
\hline 34,9 & 3,84 & 0,83 & 3,73 & 0,77 & 3,24 & 0,73 \\
\hline 38,6 & 3,91 & 0,85 & 3,83 & 0,76 & 3,32 & 0,73 \\
\hline 42,6 & 4,00 & 0,86 & 3,95 & 0,76 & 3,42 & 0,76 \\
\hline 46,6 & 4,10 & 0,87 & 4,08 & 0,76 & 3,52 & 0,77 \\
\hline 48,9 & 4,24 & 0,91 & 4,34 & 0,72 & 3,73 & 0,85 \\
\hline 52,9 & 4,34 & 0,92 & 4,46 & 0,72 & 3,85 & 0,85 \\
\hline 56,9 & 4,42 & 0,93 & 4,56 & 0,72 & 3,95 & 0,84 \\
\hline 61,2 & 4,69 & 1,04 & 5,10 & 0,83 & 4,42 & 0,82 \\
\hline 65,2 & 4,76 & 1,03 & 5,18 & 0,83 & 4,49 & 0,82 \\
\hline 69,1 & 4,80 & 1,03 & 5,23 & 0,82 & 4,55 & 0,83 \\
\hline 73,0 & 4,83 & 1,03 & 5,28 & 0,82 & 4,59 & 0,84 \\
\hline 77,0 & 4,86 & 1,03 & 5,31 & 0,82 & 4,62 & 0,85 \\
\hline 80,9 & 4,88 & 1,03 & 5,34 & 0,82 & 4,65 & 0,86 \\
\hline 84,8 & 4,90 & 1,03 & 5,36 & 0,82 & 4,67 & 0,86 \\
\hline 88,8 & 4,91 & 1,03 & 5,38 & 0,83 & 4,69 & 0,87 \\
\hline 92,7 & 4,92 & 1,03 & 5,40 & 0,83 & 4,71 & 0,87 \\
\hline 96,6 & 4,94 & 1,03 & 5,42 & 0,83 & 4,72 & 0,87 \\
\hline 100,6 & 4,95 & 1,03 & 5,44 & 0,83 & 4,74 & 0,88 \\
\hline 104,5 & 4,96 & 1,03 & 5,45 & 0,83 & 4,75 & 0,88 \\
\hline 108,5 & 5,03 & 1,04 & 5,54 & 0,87 & 4,86 & 0,91 \\
\hline Total de plantas & & 132 & & 93 & & 44 \\
\hline
\end{tabular}


Análises dos indivíduos na presença de composto orgânico

Como no modelo sem composto orgânico, também se observa que foram retidos três fatores, com autovalores maiores que um e que, juntos, explicam cerca de $94,3 \%$ da variabilidade total, o que permitiu a redução da dimensão original para três dimensões (Tabela 5).

Segundo Furtado et al. (2003), as variáveis que apresentaram altos carregamentos fatoriais num mesmo fator podem ser agrupadas, procurando-se estabelecer alguma relação técnica que justifique o agrupamento, uma vez que a interpretação do agrupamento das variáveis é subjetiva.

Tabela 4. Médias e desvios padrões das alturas da Leucaena leucocephala (Lam.) de Wit, em agrupamentos com 9 grupos, de acordo com a idade, para o tratamento sem composto orgânico.

Table 4. Mean and standard deviation for height in Leucaena leucocephala (Lam.) de Wit, clustering for nine groups, according to the age in treatment without organic compound.

\begin{tabular}{|c|c|c|c|c|c|c|c|c|c|c|}
\hline \multirow{2}{*}{$\begin{array}{c}\text { Idade } \\
\text { (meses) }\end{array}$} & \multicolumn{2}{|c|}{ Grupo 1} & \multicolumn{2}{|c|}{ Grupo 2} & \multicolumn{2}{|c|}{ Grupo 3} & \multicolumn{2}{|c|}{ Grupo 4} & \multicolumn{2}{|c|}{ Grupo 5} \\
\hline & Média & $\begin{array}{l}\text { Desvio } \\
\text { padrão }\end{array}$ & Média & $\begin{array}{l}\text { Desvio } \\
\text { padrão }\end{array}$ & Média & $\begin{array}{l}\text { Desvio } \\
\text { padrão }\end{array}$ & Média & $\begin{array}{l}\text { Desvio } \\
\text { padrão }\end{array}$ & Média & $\begin{array}{l}\text { Desvio } \\
\text { padrão }\end{array}$ \\
\hline 0,4 & 0,68 & 0,16 & 0,29 & 0,12 & 0,89 & 0,12 & 1,03 & 0,13 & 0,74 & 0,14 \\
\hline 1,2 & 0,73 & 0,15 & 0,31 & 0,13 & 0,96 & 0,07 & 1,07 & 0,11 & 0,81 & 0,10 \\
\hline 2,2 & 0,76 & 0,14 & 0,32 & 0,13 & 1,01 & 0,06 & 1,11 & 0,11 & 0,85 & 0,10 \\
\hline 3,3 & 0,83 & 0,17 & 0,33 & 0,12 & 1,18 & 0,15 & 1,17 & 0,09 & 0,89 & 0,11 \\
\hline 4,3 & 1,07 & 0,28 & 0,38 & 0,14 & 1,59 & 0,28 & 1,39 & 0,16 & 1,05 & 0,19 \\
\hline 5,3 & 1,26 & 0,34 & 0,44 & 0,18 & 1,95 & 0,32 & 1,58 & 0,21 & 1,21 & 0,26 \\
\hline 6,3 & 1,61 & 0,41 & 0,63 & 0,21 & 2,56 & 0,34 & 2,01 & 0,28 & 1,57 & 0,34 \\
\hline 7,3 & 1,92 & 0,44 & 0,96 & 0,36 & 2,99 & 0,29 & 2,41 & 0,32 & 1,87 & 0,37 \\
\hline 8,5 & 2,16 & 0,50 & 1,13 & 0,43 & 3,31 & 0,28 & 2,74 & 0,37 & 2,13 & 0,40 \\
\hline 9,6 & 2,20 & 0,50 & 1,23 & 0,39 & 3,37 & 0,31 & 2,81 & 0,39 & 2,21 & 0,39 \\
\hline 10,6 & 2,22 & 0,50 & 1,23 & 0,39 & 3,43 & 0,32 & 2,83 & 0,40 & 2,24 & 0,39 \\
\hline 12,0 & 2,29 & 0,52 & 1,40 & 0,47 & 3,48 & 0,29 & 2,93 & 0,37 & 2,33 & 0,37 \\
\hline 16,0 & 2,36 & 0,54 & 1,53 & 0,47 & 3,63 & 0,34 & 3,03 & 0,36 & 2,46 & 0,39 \\
\hline 19,0 & 2,59 & 0,64 & 1,84 & 0,54 & 4,27 & 0,55 & 3,32 & 0,39 & 2,90 & 0,55 \\
\hline 23,1 & 2,85 & 0,64 & 2,13 & 0,54 & 4,55 & 0,56 & 3,59 & 0,37 & 3,17 & 0,52 \\
\hline 27,2 & 3,03 & 0,64 & 2,32 & 0,44 & 4,78 & 0,60 & 3,79 & 0,36 & 3,37 & 0,49 \\
\hline 31,2 & 3,23 & 0,66 & 3,10 & 0,27 & 5,01 & 0,65 & 4,00 & 0,37 & 3,74 & 0,53 \\
\hline 34,9 & 3,37 & 0,66 & 3,28 & 0,27 & 5,17 & 0,64 & 4,14 & 0,37 & 3,89 & 0,51 \\
\hline 38,6 & 3,44 & 0,66 & 3,43 & 0,29 & 5,27 & 0,60 & 4,21 & 0,36 & 4,00 & 0,49 \\
\hline 42,6 & 3,53 & 0,67 & 3,50 & 0,26 & 5,38 & 0,51 & 4,29 & 0,37 & 4,15 & 0,48 \\
\hline 46,6 & 3,64 & 0,67 & 3,64 & 0,26 & 5,52 & 0,51 & 4,39 & 0,37 & 4,28 & 0,47 \\
\hline 48,9 & 3,78 & 0,68 & 3,89 & 0,31 & 5,81 & 0,36 & 4,58 & 0,39 & 4,57 & 0,43 \\
\hline 52,9 & 3,88 & 0,69 & 4,00 & 0,31 & 5,92 & 0,36 & 4,69 & 0,39 & 4,68 & 0,42 \\
\hline 56,9 & 3,96 & 0,69 & 4,11 & 0,31 & 6,03 & 0,36 & 4,79 & 0,40 & 4,78 & 0,42 \\
\hline 61,2 & 4,27 & 0,71 & 4,31 & 0,32 & 6,49 & 0,70 & 5,28 & 0,45 & 5,41 & 0,48 \\
\hline 65,2 & 4,34 & 0,71 & 4,39 & 0,31 & 6,57 & 0,69 & 5,35 & 0,45 & 5,49 & 0,48 \\
\hline 69,1 & 4,39 & 0,71 & 4,44 & 0,30 & 6,61 & 0,69 & 5,41 & 0,46 & 5,54 & 0,47 \\
\hline 73,0 & 4,42 & 0,71 & 4,47 & 0,30 & 6,63 & 0,68 & 5,45 & 0,46 & 5,59 & 0,47 \\
\hline 77,0 & 4,45 & 0,71 & 4,49 & 0,31 & 6,65 & 0,67 & 5,48 & 0,47 & 5,62 & 0,47 \\
\hline 80,9 & 4,47 & 0,71 & 4,51 & 0,32 & 6,67 & 0,66 & 5,50 & 0,48 & 5,65 & 0,47 \\
\hline 84,8 & 4,49 & 0,71 & 4,53 & 0,33 & 6,68 & 0,66 & 5,53 & 0,49 & 5,68 & 0,47 \\
\hline 88,8 & 4,51 & 0,72 & 4,54 & 0,34 & 6,70 & 0,66 & 5,55 & 0,49 & 5,70 & 0,47 \\
\hline 92,7 & 4,52 & 0,72 & 4,56 & 0,35 & 6,71 & 0,66 & 5,56 & 0,50 & 5,72 & 0,47 \\
\hline 96,6 & 4,54 & 0,72 & 4,57 & 0,36 & 6,72 & 0,65 & 5,58 & 0,50 & 5,74 & 0,47 \\
\hline 100,6 & 4,55 & 0,72 & 4,58 & 0,37 & 6,73 & 0,65 & 5,60 & 0,51 & 5,75 & 0,47 \\
\hline 104,5 & 4,56 & 0,72 & 4,60 & 0,37 & 6,74 & 0,65 & 5,61 & 0,51 & 5,77 & 0,47 \\
\hline 108,5 & 4,65 & 0,78 & 4,63 & 0,40 & 6,92 & 0,79 & 5,68 & 0,57 & 5,83 & 0,46 \\
\hline $\begin{array}{l}\text { Total de } \\
\text { plantas }\end{array}$ & \multicolumn{2}{|c|}{91} & \multicolumn{2}{|c|}{4} & \multicolumn{2}{|c|}{8} & \multicolumn{2}{|c|}{40} & \multicolumn{2}{|c|}{54} \\
\hline
\end{tabular}




\begin{tabular}{|c|c|c|c|c|c|c|c|c|}
\hline \multirow{2}{*}{$\begin{array}{l}\text { Idade } \\
\text { (meses) }\end{array}$} & \multicolumn{2}{|c|}{ Grupo 6} & \multicolumn{2}{|c|}{ Grupo 7} & \multicolumn{2}{|c|}{ Grupo 8} & \multicolumn{2}{|c|}{ Grupo 9} \\
\hline & Média & $\begin{array}{l}\text { Desvio } \\
\text { padrão }\end{array}$ & Média & $\begin{array}{l}\text { Desvio } \\
\text { padrão }\end{array}$ & Média & $\begin{array}{l}\text { Desvio } \\
\text { padrão }\end{array}$ & Média & $\begin{array}{l}\text { Desvio } \\
\text { padrão }\end{array}$ \\
\hline 0,4 & 0,37 & 0,12 & 0,87 & 0,10 & 0,95 & 0,15 & 0,71 & 0,12 \\
\hline 1,2 & 0,42 & 0,10 & 0,90 & 0,11 & 1,08 & 0,12 & 0,79 & 0,11 \\
\hline 2,2 & 0,48 & 0,09 & 0,92 & 0,11 & 1,17 & 0,15 & 0,86 & 0,09 \\
\hline 3,3 & 0,58 & 0,16 & 0,95 & 0,11 & 1,34 & 0,14 & 1,09 & 0,13 \\
\hline 4,3 & 0,82 & 0,24 & 1,01 & 0,13 & 1,77 & 0,17 & 1,58 & 0,19 \\
\hline 5,3 & 1,16 & 0,33 & 1,08 & 0,14 & 2,13 & 0,32 & 2,00 & 0,22 \\
\hline 6,3 & 1,75 & 0,31 & 1,30 & 0,22 & 2,66 & 0,42 & 2,62 & 0,27 \\
\hline 7,3 & 2,16 & 0,21 & 1,51 & 0,31 & 3,20 & 0,50 & 3,17 & 0,24 \\
\hline 8,5 & 2,46 & 0,29 & 1,62 & 0,36 & 3,53 & 0,62 & 3,46 & 0,24 \\
\hline 9,6 & 2,57 & 0,27 & 1,65 & 0,35 & 3,61 & 0,61 & 3,54 & 0,26 \\
\hline 10,6 & 2,61 & 0,28 & 1,67 & 0,35 & 3,66 & 0,64 & 3,58 & 0,25 \\
\hline 12,0 & 2,67 & 0,30 & 1,69 & 0,36 & 3,72 & 0,62 & 3,65 & 0,25 \\
\hline 16,0 & 2,79 & 0,32 & 1,76 & 0,36 & 3,76 & 0,64 & 3,70 & 0,25 \\
\hline 19,0 & 3,11 & 0,39 & 1,87 & 0,38 & 3,90 & 0,70 & 3,83 & 0,32 \\
\hline 23,1 & 3,38 & 0,40 & 2,14 & 0,38 & 4,14 & 0,71 & 4,09 & 0,31 \\
\hline 27,2 & 3,60 & 0,43 & 2,29 & 0,41 & 4,29 & 0,69 & 4,27 & 0,31 \\
\hline 31,2 & 3,97 & 0,71 & 2,51 & 0,52 & 4,43 & 0,68 & 4,39 & 0,33 \\
\hline 34,9 & 4,15 & 0,71 & 2,63 & 0,53 & 4,56 & 0,69 & 4,55 & 0,34 \\
\hline 38,6 & 4,27 & 0,67 & 2,70 & 0,55 & 4,57 & 0,68 & 4,65 & 0,35 \\
\hline 42,6 & 4,55 & 0,63 & 2,74 & 0,56 & 4,59 & 0,68 & 4,75 & 0,37 \\
\hline 46,6 & 4,68 & 0,63 & 2,84 & 0,56 & 4,68 & 0,70 & 4,86 & 0,36 \\
\hline 48,9 & 4,94 & 0,61 & 3,04 & 0,56 & 4,77 & 0,72 & 5,05 & 0,40 \\
\hline 52,9 & 5,05 & 0,61 & 3,14 & 0,56 & 4,88 & 0,72 & 5,16 & 0,40 \\
\hline 56,9 & 5,15 & 0,60 & 3,22 & 0,57 & 4,96 & 0,72 & 5,25 & 0,40 \\
\hline 61,2 & 5,64 & 0,26 & 3,47 & 0,63 & 5,18 & 0,60 & 5,54 & 0,48 \\
\hline 65,2 & 5,71 & 0,25 & 3,54 & 0,63 & 5,25 & 0,60 & 5,61 & 0,47 \\
\hline 69,1 & 5,77 & 0,24 & 3,59 & 0,63 & 5,29 & 0,60 & 5,66 & 0,47 \\
\hline 73,0 & 5,80 & 0,23 & 3,63 & 0,64 & 5,32 & 0,60 & 5,69 & 0,46 \\
\hline 77,0 & 5,83 & 0,21 & 3,65 & 0,65 & 5,34 & 0,59 & 5,72 & 0,46 \\
\hline 80,9 & 5,86 & 0,20 & 3,68 & 0,65 & 5,36 & 0,59 & 5,73 & 0,45 \\
\hline 84,8 & 5,88 & 0,19 & 3,69 & 0,65 & 5,38 & 0,58 & 5,75 & 0,45 \\
\hline 88,8 & 5,89 & 0,19 & 3,71 & 0,66 & 5,39 & 0,58 & 5,77 & 0,45 \\
\hline 92,7 & 5,90 & 0,19 & 3,73 & 0,66 & 5,41 & 0,58 & 5,78 & 0,45 \\
\hline 96,6 & 5,92 & 0,19 & 3,74 & 0,66 & 5,42 & 0,58 & 5,80 & 0,45 \\
\hline 100,6 & 5,93 & 0,19 & 3,75 & 0,66 & 5,44 & 0,58 & 5,81 & 0,45 \\
\hline 104,5 & 5,94 & 0,19 & 3,77 & 0,67 & 5,45 & 0,58 & 5,82 & 0,45 \\
\hline 108,5 & 5,97 & 0,20 & 3,91 & 0,78 & 5,53 & 0,58 & 5,87 & 0,45 \\
\hline $\begin{array}{l}\text { Total de } \\
\text { plantas }\end{array}$ & \multicolumn{2}{|c|}{7} & \multicolumn{2}{|c|}{34} & \multicolumn{2}{|c|}{7} & \multicolumn{2}{|c|}{24} \\
\hline
\end{tabular}

Observa-se que o fator 1 possui correlação acima de 0,7 com medições de altura da $20^{\mathrm{a}}$ à $37^{\mathrm{a}}$ medição, indicando que esse fator expressa o crescimento da leucena do $7^{\circ}$ mês do $4^{\circ}$ ano ao $12^{\circ}$ mês do $9^{\circ}$ ano após o plantio, com $73,91 \%$ de explicação do total da variância.

$\mathrm{O}$ fator 2 tem uma explicação de $14,34 \%$ da variância da $5^{\mathrm{a}}$ à $19^{\mathrm{a}}$ medição, ou seja, o comportamento da leucena nesse fator descreve o crescimento em altura do $5^{\circ}$ mês do $1^{\circ}$ ano ao $6^{\circ}$ mês do $4^{\circ}$ ano.

O fator 3 tem alta explicação da $1^{a}$ à $4^{a}$ medição, com 5,93\% de explicação da variância. As leucenas têm crescimento ao longo do tempo representado por esse fator do $1^{\circ}$ ao $4^{\circ}$ mês do $1^{\circ}$ ano após o plantio.

Para Souza et al. (1997), há métodos que podem ser adotados para se determinar o número de grupos. Todavia, o que se faz comumente é utilizar vários números de grupos e, por algum critério de otimização, selecionar o mais conveniente, como, por exemplo, a análise discriminante, usada neste estudo para verificação da adequação da partição obtida. 

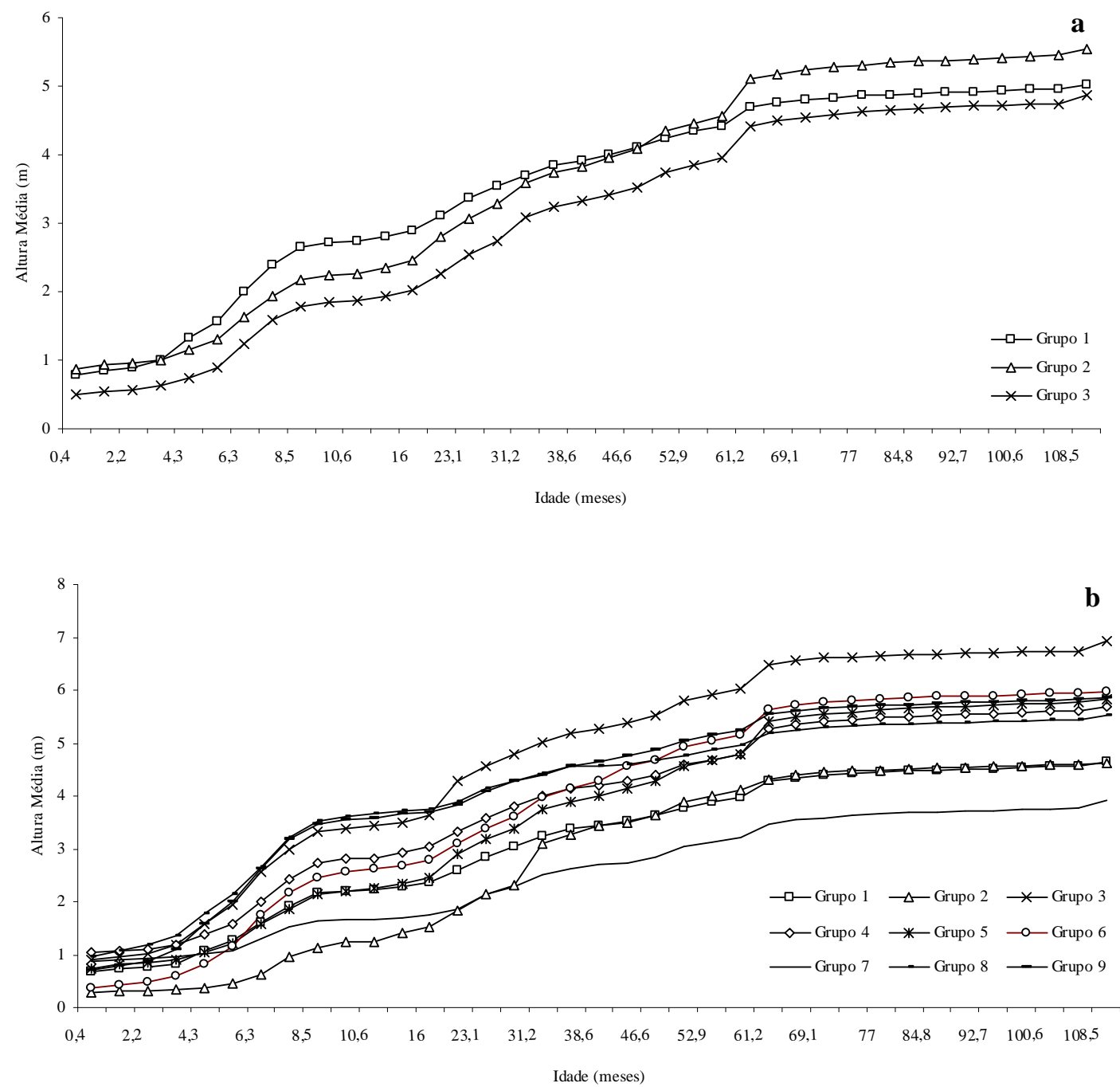

Figura 1. Crescimento em altura da Leucaena leucocephala (Lam.) de Wit ao longo do tempo, no tratamento sem composto orgânico, nos agrupamentos com 3(a) e 9(b) grupos.

Figure 1. Height growth of the Leucaena leucocephala (Lam.) de Wit along the time, in treatment without organic compound for clustering on third (a) and nineth (b) groups.

Com a aplicação da análise discriminante, observou-se que a melhor classificação de grupos está no agrupamento com 10 grupos, resultando em $95,1 \%$ de percentual de classificação correta das leucenas em cada agrupamento, dos oito modelos gerados pela análise discriminante. Em seguida, os agrupamentos com 3 e 5 grupos tiveram percentual de classificação equivalente a 94,7\% (Tabela 6).

Nas plantas que receberam composto orgânico, observa-se que na fase inicial de crescimento, os grupos 1 e 8 tiveram as menores variações de altura, de $0,50 \mathrm{~m}$ a $0,82 \mathrm{~m}$ e de $0,46 \mathrm{~m}$ a $0,65 \mathrm{~m}$, respectivamente. Entre os que apresentaram maior crescimento, o grupo 4 variou na média entre $1,16 \mathrm{~m} \mathrm{e}$ $1,37 \mathrm{~m}$, do $1^{\circ}$ mês ao $3^{\circ}$ mês do $1^{\circ}$ ano após o plantio, em 7 plantas agrupadas (Tabela 7).

Do $4^{\circ}$ mês do $1^{\circ}$ ano ao $3^{\circ}$ mês do $2^{\circ}$ ano, que representa a $2^{\mathrm{a}}$ fase (período de 4,3 a 38,6 meses de crescimento da planta), 15 plantas do grupo 10 tiveram alturas variando de $1,77 \mathrm{~m}$ a $5,06 \mathrm{~m}$, em média. O grupo de menor crescimento nessa fase foi o grupo 2 , variando de $0,85 \mathrm{~m}$ a $2,39 \mathrm{~m}$, com 4 plantas agrupadas. 
Tabela 5. Matriz de cargas fatoriais, comunalidades e variâncias específicas segundo a idade da Leucaena leucocephala (Lam.) de Wit, obtidos na rotação varimax, tratamento com composto.

Table 5. Matrix of factoid loadings, communalities and specific variance, according to the age of Leucena leucocephala (Lam.) de Wit, obtained in varimax rotation for treatment with organic compound.

\begin{tabular}{|c|c|c|c|c|c|}
\hline \multirow{2}{*}{ Idade (meses) } & \multicolumn{3}{|c|}{ Fator } & \multirow{2}{*}{ Comunalidade } & \multirow{2}{*}{$\begin{array}{l}\text { Variância } \\
\text { específica }\end{array}$} \\
\hline & 1 & 2 & 3 & & \\
\hline 0,4 & $-0,0930$ & 0,0672 & 0,9430 & 0,9024 & 0,0976 \\
\hline 1,2 & $-0,0240$ & 0,1864 & 0,9609 & 0,9586 & 0,0414 \\
\hline 2,2 & 0,0228 & 0,3324 & 0,9196 & 0,9568 & 0,0432 \\
\hline 3,3 & 0,1388 & 0,6281 & 0,6988 & 0,9021 & 0,0979 \\
\hline 4,3 & 0,2046 & 0,8123 & 0,4277 & 0,8845 & 0,1155 \\
\hline 5,3 & 0,2470 & $\mathbf{0 , 8 5 5 7}$ & 0,3095 & 0,8890 & 0,1110 \\
\hline 6,3 & 0,2897 & 0,8720 & 0,2499 & 0,9068 & 0,0932 \\
\hline 7,3 & 0,3241 & 0,8935 & 0,1956 & 0,9417 & 0,0583 \\
\hline 8,5 & 0,3509 & 0,8948 & 0,1775 & 0,9554 & 0,0446 \\
\hline 9,6 & 0,3607 & 0,8964 & 0,1559 & 0,9580 & 0,0420 \\
\hline 10,6 & 0,3613 & $\mathbf{0 , 8 9 7 3}$ & 0,1591 & 0,9611 & 0,0389 \\
\hline 12,0 & 0,3757 & 0,8962 & 0,1475 & 0,9660 & 0,0340 \\
\hline 16,0 & 0,4122 & 0,8780 & 0,1323 & 0,9582 & 0,0418 \\
\hline 19,0 & 0,5063 & 0,8008 & 0,1033 & 0,9083 & 0,0917 \\
\hline 23,1 & 0,5263 & 0,7961 & 0,0918 & 0,9192 & 0,0808 \\
\hline 27,2 & 0,5618 & $\mathbf{0 , 7 8 1 9}$ & 0,0643 & 0,9310 & 0,0690 \\
\hline 31,2 & 0,6192 & 0,7089 & $-0,0317$ & 0,8870 & 0,1130 \\
\hline 34,9 & 0,6435 & 0,6964 & $-0,0287$ & 0,8998 & 0,1002 \\
\hline 38,6 & 0,6710 & 0,6791 & $-0,0240$ & 0,9119 & 0,0881 \\
\hline 42,6 & 0,7149 & 0,6317 & $-0,0082$ & 0,9101 & 0,0899 \\
\hline 46,6 & 0,7222 & 0,6243 & $-0,0061$ & 0,9114 & 0,0886 \\
\hline 48,9 & 0,7795 & 0,5377 & 0,0021 & 0,8968 & 0,1032 \\
\hline 52,9 & 0,7827 & 0,5356 & 0,0024 & 0,8995 & 0,1005 \\
\hline 56,9 & 0,7914 & 0,5270 & $-0,0006$ & 0,9040 & 0,0960 \\
\hline 61,2 & 0,9305 & 0,3342 & 0,0419 & 0,9793 & 0,0207 \\
\hline 65,2 & 0,9320 & 0,3344 & 0,0419 & 0,9823 & 0,0177 \\
\hline 69,1 & 0,9356 & 0,3304 & 0,0419 & 0,9862 & 0,0138 \\
\hline 73,0 & 0,9389 & 0,3258 & 0,0401 & 0,9892 & 0,0108 \\
\hline 77,0 & 0,9418 & 0,3203 & 0,0375 & 0,9910 & 0,0090 \\
\hline 80,9 & 0,9440 & 0,3148 & 0,0350 & 0,9915 & 0,0085 \\
\hline 84,8 & 0,9456 & 0,3097 & 0,0326 & 0,9911 & 0,0089 \\
\hline 88,8 & 0,9465 & 0,3051 & 0,0307 & 0,9899 & 0,0101 \\
\hline 92,7 & 0,9470 & 0,3018 & 0,0295 & 0,9887 & 0,0113 \\
\hline 96,6 & 0,9471 & 0,2987 & 0,0284 & 0,9871 & 0,0129 \\
\hline 100,6 & 0,9471 & 0,2957 & 0,0273 & 0,9853 & 0,0147 \\
\hline 104,5 & 0,9469 & 0,2933 & 0,0263 & 0,9834 & 0,0166 \\
\hline 108,5 & 0,9266 & 0,2634 & $-0,0002$ & 0,9280 & 0,0720 \\
\hline \% Variância explicada & 73,7611 & 14,6484 & 5,8944 & - & - \\
\hline
\end{tabular}

Para a fase que corresponde à idade de 42,6 meses a 108,5 meses $\left(7^{\circ}\right.$ mês do $3^{\circ}$ ano ao $12^{\circ}$ mês do $9^{\circ}$ ano da planta), percebe-se que o grupo 2 é o que possui menor crescimento em altura, de $2,40 \mathrm{~m}$ a $3,09 \mathrm{~m}$ na $37^{\mathrm{a}}$ medição. Entre as maiores alturas observadas, temos os grupos 1 e 9, com medições estimadas de 4,57 a 6,30 m e de 4,04 $\mathrm{m}$ a 6,23 m, respectivamente.

No tratamento sem o composto orgânico nos 9 grupos predeterminados, o crescimento em altura, em média, foi superior ao tratamento que recebeu composto orgânico, atingindo $6,92 \mathrm{~m}$ no último mês do $9^{\circ}$ ano do plantio, a 108,5 meses de idade (Figura 2). 
Tabela 6. Resultado da análise discriminante, segundo percentual de classificação correta dos grupos, para a Leucaena leucocephala (Lam.) de Wit, no tratamento com composto orgânico.

Table 6. Results from discriminant analysis, according to the correct percent classification of groups, for Leucaena leucocephala (Lam.) de Wit, in treatment with organic compound.

\begin{tabular}{lc}
\hline Número de grupos & Percentual de classificação correta \\
\hline 3 & $94,7 \%$ \\
4 & $93,2 \%$ \\
5 & $94,7 \%$ \\
6 & $92,5 \%$ \\
7 & $92,1 \%$ \\
8 & $94,3 \%$ \\
9 & $89,4 \%$ \\
$\mathbf{1 0}$ & $\mathbf{9 5 , 1 \%}$ \\
\hline
\end{tabular}

Tabela 7. Médias e desvios padrões das alturas da Leucaena leucocephala (Lam.) de Wit, em agrupamentos com 10 grupos, de acordo com a idade, para o tratamento com composto orgânico.

Table 7. Mean and standard deviation for height in Leucaena leucocephala (Lam.) de Wit, clustering for ten groups, according to the age in treatment without compound residue organic.

\begin{tabular}{|c|c|c|c|c|c|c|c|c|c|c|}
\hline \multirow[b]{2}{*}{$\begin{array}{l}\text { Idade } \\
\text { (meses) }\end{array}$} & \multicolumn{2}{|c|}{ Grupo 1} & \multicolumn{2}{|c|}{ Grupo 2 } & \multicolumn{2}{|c|}{ Grupo 3} & \multicolumn{2}{|c|}{ Grupo 4} & \multicolumn{2}{|c|}{ Grupo 5} \\
\hline & Média & $\begin{array}{l}\text { Desvio } \\
\text { padrão }\end{array}$ & Média & $\begin{array}{l}\text { Desvio } \\
\text { padrão }\end{array}$ & Média & $\begin{array}{l}\text { Desvio } \\
\text { padrão }\end{array}$ & Média & $\begin{array}{l}\text { Desvio } \\
\text { padrão }\end{array}$ & Média & $\begin{array}{l}\text { Desvio } \\
\text { padrão }\end{array}$ \\
\hline 0,4 & 0,50 & 0,09 & 0,63 & 0,06 & 0,85 & 0,12 & 1,16 & 0,08 & 0,92 & 0,10 \\
\hline 1,2 & 0,59 & 0,09 & 0,65 & 0,07 & 0,91 & 0,11 & 1,23 & 0,08 & 0,98 & 0,09 \\
\hline 2,2 & 0,67 & 0,09 & 0,68 & 0,07 & 0,96 & 0,11 & 1,29 & 0,10 & 1,05 & 0,11 \\
\hline 3,3 & 0,82 & 0,20 & 0,71 & 0,07 & 1,11 & 0,15 & 1,37 & 0,13 & 1,22 & 0,16 \\
\hline 4,3 & 1,10 & 0,29 & 0,85 & 0,21 & 1,47 & 0,24 & 1,68 & 0,19 & 1,65 & 0,26 \\
\hline 5,3 & 1,38 & 0,35 & 0,94 & 0,27 & 1,75 & 0,30 & 1,95 & 0,28 & 1,98 & 0,40 \\
\hline 6,3 & 1,83 & 0,45 & 1,12 & 0,34 & 2,24 & 0,38 & 2,48 & 0,32 & 2,50 & 0,48 \\
\hline 7,3 & 2,21 & 0,44 & 1,26 & 0,46 & 2,64 & 0,43 & 2,85 & 0,26 & 2,94 & 0,53 \\
\hline 8,5 & 2,51 & 0,44 & 1,36 & 0,50 & 2,94 & 0,45 & 3,11 & 0,29 & 3,26 & 0,49 \\
\hline 9,6 & 2,59 & 0,46 & 1,41 & 0,49 & 3,00 & 0,46 & 3,15 & 0,30 & 3,32 & 0,48 \\
\hline 10,6 & 2,61 & 0,46 & 1,43 & 0,51 & 3,03 & 0,46 & 3,22 & 0,32 & 3,35 & 0,49 \\
\hline 12,0 & 2,71 & 0,43 & 1,45 & 0,53 & 3,12 & 0,45 & 3,28 & 0,30 & 3,46 & 0,53 \\
\hline 16,0 & 2,81 & 0,41 & 1,49 & 0,52 & 3,23 & 0,45 & 3,37 & 0,32 & 3,51 & 0,54 \\
\hline 19,0 & 3,26 & 0,34 & 1,73 & 0,38 & 3,50 & 0,42 & 3,69 & 0,45 & 3,67 & 0,59 \\
\hline 23,1 & 3,54 & 0,34 & 2,01 & 0,38 & 3,77 & 0,42 & 3,95 & 0,44 & 3,89 & 0,55 \\
\hline 27,2 & 3,78 & 0,33 & 2,17 & 0,38 & 3,95 & 0,41 & 4,09 & 0,45 & 3,98 & 0,53 \\
\hline 31,2 & 4,28 & 0,34 & 2,30 & 0,44 & 4,14 & 0,43 & 4,16 & 0,44 & 4,06 & 0,50 \\
\hline 34,9 & 4,42 & 0,34 & 2,36 & 0,48 & 4,28 & 0,42 & 4,32 & 0,42 & 4,17 & 0,50 \\
\hline 38,6 & 4,50 & 0,34 & 2,39 & 0,52 & 4,35 & 0,41 & 4,43 & 0,41 & 4,21 & 0,51 \\
\hline 42,6 & 4,57 & 0,37 & 2,40 & 0,53 & 4,44 & 0,41 & 4,54 & 0,42 & 4,22 & 0,51 \\
\hline 46,6 & 4,66 & 0,38 & 2,42 & 0,54 & 4,55 & 0,41 & 4,63 & 0,43 & 4,32 & 0,52 \\
\hline 48,9 & 4,92 & 0,48 & 2,43 & 0,54 & 4,75 & 0,49 & 4,87 & 0,48 & 4,38 & 0,51 \\
\hline 52,9 & 5,03 & 0,48 & 2,52 & 0,54 & 4,86 & 0,49 & 4,98 & 0,48 & 4,47 & 0,51 \\
\hline 56,9 & 5,15 & 0,51 & 2,57 & 0,53 & 4,95 & 0,49 & 5,07 & 0,49 & 4,54 & 0,52 \\
\hline 61,2 & 5,73 & 0,41 & 2,58 & 0,53 & 5,36 & 0,53 & 5,60 & 0,39 & 4,65 & 0,58 \\
\hline 65,2 & 5,81 & 0,41 & 2,63 & 0,51 & 5,43 & 0,53 & 5,67 & 0,40 & 4,72 & 0,58 \\
\hline 69,1 & 5,87 & 0,41 & 2,66 & 0,51 & 5,48 & 0,53 & 5,72 & 0,40 & 4,77 & 0,58 \\
\hline 73,0 & 5,92 & 0,41 & 2,70 & 0,51 & 5,52 & 0,53 & 5,77 & 0,40 & 4,81 & 0,59 \\
\hline 77,0 & 5,96 & 0,40 & 2,73 & 0,52 & 5,55 & 0,53 & 5,80 & 0,41 & 4,84 & 0,59 \\
\hline 80,9 & 6,00 & 0,40 & 2,75 & 0,52 & 5,58 & 0,52 & 5,82 & 0,41 & 4,86 & 0,58 \\
\hline 84,8 & 6,03 & 0,39 & 2,78 & 0,53 & 5,60 & 0,52 & 5,84 & 0,41 & 4,88 & 0,58 \\
\hline 88,8 & 6,06 & 0,39 & 2,79 & 0,54 & 5,62 & 0,52 & 5,85 & 0,41 & 4,90 & 0,58 \\
\hline 92,7 & 6,08 & 0,39 & 2,81 & 0,54 & 5,64 & 0,53 & 5,87 & 0,42 & 4,91 & 0,58 \\
\hline 96,6 & 6,10 & 0,39 & 2,82 & 0,55 & 5,66 & 0,53 & 5,88 & 0,42 & 4,93 & 0,58 \\
\hline 100,6 & 6,12 & 0,39 & 2,84 & 0,56 & 5,67 & 0,53 & 5,89 & 0,42 & 4,94 & 0,58 \\
\hline 104,5 & 6,14 & 0,39 & 2,85 & 0,56 & 5,69 & 0,53 & 5,91 & 0,42 & 4,95 & 0,58 \\
\hline 108,5 & 6,30 & 0,50 & 3,09 & 0,92 & 5,76 & 0,56 & 5,96 & 0,46 & 5,02 & 0,63 \\
\hline $\begin{array}{l}\text { Total de } \\
\text { plantas }\end{array}$ & & \multicolumn{2}{|c|}{4} & \multicolumn{2}{|c|}{124} & & \\
\hline
\end{tabular}




\begin{tabular}{|c|c|c|c|c|c|c|c|c|c|c|}
\hline \multirow{2}{*}{$\begin{array}{l}\text { Idade } \\
\text { (meses) }\end{array}$} & \multicolumn{2}{|c|}{ Grupo 6} & \multicolumn{2}{|c|}{ Grupo 7} & \multicolumn{2}{|c|}{ Grupo 8} & \multicolumn{2}{|c|}{ Grupo 9} & \multicolumn{2}{|c|}{ Grupo 10} \\
\hline & Média & $\begin{array}{l}\text { Desvio } \\
\text { padrão }\end{array}$ & Média & $\begin{array}{l}\text { Desvio } \\
\text { padrão }\end{array}$ & Média & $\begin{array}{l}\text { Desvio } \\
\text { padrão }\end{array}$ & Média & $\begin{array}{l}\text { Desvio } \\
\text { padrão }\end{array}$ & Média & $\begin{array}{l}\text { Desvio } \\
\text { padrão }\end{array}$ \\
\hline 0,4 & 0,96 & 0,09 & 0,62 & 0,13 & 0,46 & 0,12 & 0,92 & 0,12 & 0,68 & 0,13 \\
\hline 1,2 & 1,00 & 0,11 & 0,65 & 0,12 & 0,50 & 0,13 & 0,96 & 0,13 & 0,80 & 0,11 \\
\hline 2,2 & 1,02 & 0,11 & 0,69 & 0,12 & 0,56 & 0,17 & 0,98 & 0,13 & 0,93 & 0,09 \\
\hline 3,3 & 1,06 & 0,12 & 0,73 & 0,12 & 0,65 & 0,25 & 1,01 & 0,14 & 1,25 & 0,17 \\
\hline 4,3 & 1,14 & 0,15 & 0,87 & 0,19 & 0,90 & 0,39 & 1,11 & 0,21 & 1,77 & 0,22 \\
\hline 5,3 & 1,25 & 0,19 & 1,01 & 0,23 & 1,12 & 0,42 & 1,22 & 0,29 & 2,24 & 0,30 \\
\hline 6,3 & 1,56 & 0,28 & 1,37 & 0,26 & 1,46 & 0,48 & 1,53 & 0,37 & 2,92 & 0,33 \\
\hline 7,3 & 1,80 & 0,32 & 1,69 & 0,20 & 1,81 & 0,36 & 1,78 & 0,39 & 3,46 & 0,38 \\
\hline 8,5 & 1,97 & 0,37 & 1,90 & 0,22 & 2,10 & 0,35 & 2,06 & 0,40 & 3,78 & 0,33 \\
\hline 9,6 & 2,02 & 0,37 & 1,95 & 0,23 & 2,17 & 0,32 & 2,14 & 0,38 & 3,87 & 0,34 \\
\hline 10,6 & 2,04 & 0,38 & 1,97 & 0,22 & 2,18 & 0,31 & 2,16 & 0,36 & 3,92 & 0,33 \\
\hline 12,0 & 2,08 & 0,40 & 2,04 & 0,25 & 2,33 & 0,29 & 2,25 & 0,32 & 3,99 & 0,34 \\
\hline 16,0 & 2,13 & 0,41 & 2,16 & 0,29 & 2,47 & 0,33 & 2,41 & 0,29 & 4,11 & 0,30 \\
\hline 19,0 & 2,27 & 0,45 & 2,40 & 0,36 & 2,68 & 0,39 & 2,92 & 0,44 & 4,32 & 0,35 \\
\hline 23,1 & 2,53 & 0,42 & 2,68 & 0,36 & 2,95 & 0,39 & 3,20 & 0,43 & 4,57 & 0,35 \\
\hline 27,2 & 2,68 & 0,43 & 2,90 & 0,37 & 3,14 & 0,40 & 3,38 & 0,38 & 4,70 & 0,40 \\
\hline 31,2 & 2,84 & 0,50 & 3,22 & 0,48 & 3,36 & 0,38 & 3,59 & 0,29 & 4,87 & 0,55 \\
\hline 34,9 & 2,94 & 0,51 & 3,37 & 0,48 & 3,49 & 0,41 & 3,76 & 0,29 & 4,99 & 0,51 \\
\hline 38,6 & 2,99 & 0,51 & 3,46 & 0,45 & 3,55 & 0,46 & 3,86 & 0,27 & 5,06 & 0,49 \\
\hline 42,6 & 3,01 & 0,51 & 3,61 & 0,42 & 3,59 & 0,52 & 4,04 & 0,31 & 5,12 & 0,47 \\
\hline 46,6 & 3,11 & 0,52 & 3,72 & 0,41 & 3,69 & 0,52 & 4,16 & 0,31 & 5,20 & 0,46 \\
\hline 48,9 & 3,23 & 0,55 & 3,97 & 0,47 & 3,78 & 0,48 & 4,45 & 0,40 & 5,28 & 0,47 \\
\hline 52,9 & 3,33 & 0,54 & 4,08 & 0,47 & 3,86 & 0,47 & 4,56 & 0,40 & 5,39 & 0,47 \\
\hline 56,9 & 3,41 & 0,54 & 4,17 & 0,47 & 3,91 & 0,46 & 4,66 & 0,40 & 5,49 & 0,47 \\
\hline 61,2 & 3,67 & 0,58 & 4,51 & 0,48 & 4,12 & 0,53 & 5,60 & 0,37 & 5,72 & 0,46 \\
\hline 65,2 & 3,74 & 0,58 & 4,58 & 0,48 & 4,19 & 0,54 & 5,66 & 0,37 & 5,79 & 0,45 \\
\hline 69,1 & 3,79 & 0,59 & 4,63 & 0,47 & 4,23 & 0,55 & 5,72 & 0,36 & 5,84 & 0,43 \\
\hline 73,0 & 3,82 & 0,59 & 4,66 & 0,48 & 4,27 & 0,55 & 5,78 & 0,36 & 5,87 & 0,43 \\
\hline 77,0 & 3,84 & 0,59 & 4,70 & 0,49 & 4,30 & 0,55 & 5,83 & 0,36 & 5,89 & 0,43 \\
\hline 80,9 & 3,85 & 0,59 & 4,72 & 0,49 & 4,32 & 0,56 & 5,87 & 0,35 & 5,90 & 0,43 \\
\hline 84,8 & 3,86 & 0,60 & 4,75 & 0,50 & 4,34 & 0,56 & 5,91 & 0,35 & 5,92 & 0,43 \\
\hline 88,8 & 3,87 & 0,60 & 4,77 & 0,50 & 4,36 & 0,57 & 5,94 & 0,35 & 5,93 & 0,43 \\
\hline 92,7 & 3,88 & 0,60 & 4,79 & 0,51 & 4,38 & 0,57 & 5,97 & 0,35 & 5,95 & 0,43 \\
\hline 96,6 & 3,89 & 0,60 & 4,80 & 0,51 & 4,40 & 0,58 & 5,99 & 0,35 & 5,96 & 0,43 \\
\hline 100,6 & 3,90 & 0,60 & 4,82 & 0,51 & 4,41 & 0,58 & 6,02 & 0,35 & 5,97 & 0,43 \\
\hline 104,5 & 3,91 & 0,60 & 4,83 & 0,52 & 4,43 & 0,59 & 6,04 & 0,35 & 5,98 & 0,43 \\
\hline 108,5 & 3,92 & 0,61 & 4,96 & 0,63 & 4,52 & 0,57 & 6,23 & 0,51 & 6,05 & 0,47 \\
\hline $\begin{array}{l}\text { Total de } \\
\text { plantas }\end{array}$ & \multicolumn{2}{|c|}{28} & \multicolumn{2}{|c|}{27} & \multicolumn{2}{|c|}{6} & \multicolumn{2}{|c|}{15} & \multicolumn{2}{|c|}{15} \\
\hline
\end{tabular}

\section{CONCLUSOES}

Com as técnicas de análise multivariada, foi possível descrever o comportamento do crescimento em altura da Leucaena leucocephala (Lam.) de Wit ao longo do tempo, na Estação Experimental de Caruaru, em PE.

Com a análise fatorial foi possível representar as fases de crescimento da leucena na presença ou não de composto orgânico. 


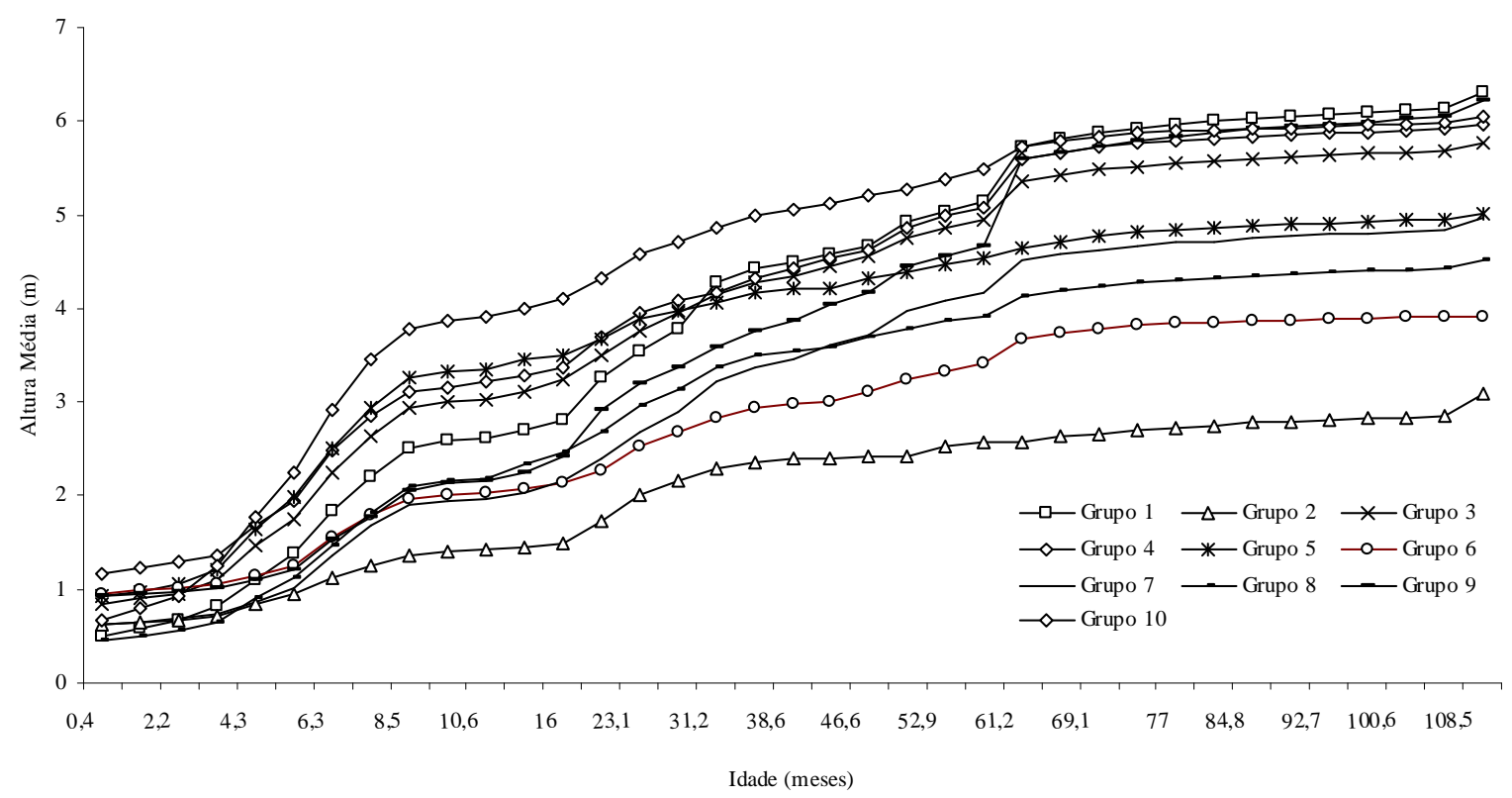

Figura 2. Crescimento em altura da Leucaena leucocephala (Lam.) de Wit, ao longo do tempo, no tratamento com composto orgânico, no agrupamento com 10 grupos.

Figure 2. Growth in height of the Leucaena leucocephala (Lam.) de Wit, along the time, in treatment without organic compound, for clustering with ten groups.

\section{REFERÊNCIAS}

AFIF, A. A.; CLARK, V. Computer-aided multivariate analysis. London: Chapman e Hall, 1996. 455 p.

ANDERSON, T. W. An introduction to multivariate statistical analysis, New York: J. Wiley \& Sons, 1984. $675 \mathrm{p}$.

BRITO, C. R. de; SILVA, J. A. A. da; FERREIRA, R. L. C.; SANTOS, E. de S.; FERRAZ, I. Modelos de crescimento resultantes da combinação e variações dos modelos de Chapman-Richards e Silva-Bailey aplicados em Leucaena leucocephala (Lam.) de Wit. Ciência Florestal, Santa Maria, v. 17, n. 2, p. 175185, abr-jun, 2007.

BURTON, A. J.; RAMM, C. W.; PREGITZER, K. S. Use of multivariate methods in forest research site selection. Canadian Journal Forest Research, Ottawa, v. 21, p. 1573-1580, 1991.

CLUTTER, J. L.; FORTSON, J. C.; PIENNAAR, L. V.; BRISTER, G. H.; BAILEY, R. L. Timber management: a quantitative approach. New York: J. Wiley \& Sons, 1983. 333 p.

DAVIS, L. S.; JOHNSON, K. N. Forest management. New York: Mc Graw-Hill, 1987. 790 p.

DRAPER, N. R.; SMITH, H. Applied regression analysis. 3. ed. New York: J. Wiley \& Sons, 1998. 736 p.

DRUMOND, M. A. Leucena - uma arbórea de uso múltiplo, para a região semi-árida do nordeste brasileiro. In: SIMPÓSIO BRASILEIRO DE CAPTAÇÃO DE ÁGUA DE CHUVA NO SEMI-ÁRIDO, 3., 2001, Petrolina. Anais... Petrolina: EMBRAPA, 2001.4 p.

FREITAS, A. R. D.; OlIVEIRA, A. L. P. C. D.; SILVA, B. A. da; DECICO, M. J. U. Leucaena leucocephala Lam. (de Wit.): cultura e melhoramento. São Carlos: Embrapa-UEPAE, 1991. 93 p. (Documento, n. 12). 
FURTADO, E. M.; CHAVES NETO, A.; DOMINGUES, Z. N.; HOSOKAWA, R. T. Ranqueamento de faxinais do Estado do Paraná através da análise fatorial. Revista Ciências Exatas e Naturais, Guarapuava, v. 5, n. 1, p. 85-98, jan./jun. 2003.

JOHNSON, R. A.; WICHERN, D. W. Applied multivariate statistical analysis. 4. ed. New Jersey: Prentice Hall, 1998, 816 p.

KAISER, H. F. The varimax criterion for analytic rotation in factor analysis. Psychometrika, Williamsburg, v. 23, n. 3, p.187-200, 1958.

MAESTRI, R.; SANQUETTA, C. R.; ARCE, J. E. Modelagem do crescimento de povoamentos de Eucalyptus grandis através de processos de difusão. Floresta, Curitiba, v. 33, n. 2, p. 169-182, 2003.

MANLY, B. F. J. Multivariate statistical methods: a primer. 2. ed. London: Chapman \& Hall, 1997. 215 p.

MENDES, B. R.; CALEGARIO, N.; VOlPATO, C. E. S.; MELlO, A. A. de. Desenvolvimento de modelos de crescimento de árvores individuais fundamentado em equações diferenciais. Cerne, Lavras, v. 12 , n. 3, p. $254-263,2006$

MEUNIER, I. M. J. Crescimento de mudas de Leucaena leucocephala (Lam.) de Wit. Em função do uso de composto de resíduo urbano, adubação fosfatada e inoculação com Rhizobium loti. $110 \mathrm{f}$. Dissertação (Mestrado em Agronomia, Ciência do Solo) - Universidade Federal Rural de Pernambuco, Recife, 1991.

NETER, J.; WASSERMAN, W.; KUTNER, M. H. Applied linear statistical models: regression, analysis of variance, experimental designs. 3. ed. Chicago: Irwing, 1996. 1127 p.

OLIVEIRA, R. M. B.; ARLINDO, D. M.; PEREIRA, I. E. Avaliação de diferentes tamanhos de sacos de polietileno sobre o desenvolvimento de mudas de Leucena (Leucaena leucocephala (Lam). de Wit). Revista de Biologia e Ciências da Terra, Campina Grande, PB, v. 4, n. 2, n. p, 2004.

ROSSI, L. M. B.; KOEHLER, H. R.; SANQUETTA, C. R.; ARCE, J. E. Modelagem de mortalidade em florestas naturais. Floresta, Curitiba, v. 37, n. 2, p. 275-291, 2007.

SCOLFORO, J. R. Mensuração florestal. módulo 4: avaliação da produtividade através da classificação do sítio. Lavras: ESAL/FAEPE, 1993. 138 p.

SEIFFERT, N. F.; THIAGO, L. R. Legumineira: cultura forrageira para a produção de proteína. Campo Grande: EMBRAPA - CNPGC. 1983. 52 p. (Circular Técnica, n. 13).

SILVA, C. M. M. de. S. de. Avaliação de gênero Leucena na região semi-árida de Pernambuco. Petrolina: EMBRAPA - CPATSA, 1992, 21 p. (Boletim de Pesquisa, n. 44).

SOUZA, A. L.; FERREIRA, R. L. C.; XAVIER, A. Análise de agrupamento aplicada à área florestal. Sociedade de investigações florestais. Viçosa, MG: SIF, 1997. 109 p. (Documento SIF, n. 16).

STATISTICAL PACKAGE FOR THE SOCIAL SCIENCES - SPSS. Command Syntax Reference. SPSS $^{\circledR}$ 13.0. Chicago, 2004. p. 593-610. 